\title{
Announcement
}

\section{Community Preventive Services Task Force Recommendation for Text Messaging Interventions to Improve Medication Adherence for Chronic Disease Management}

The Community Preventive Services Task Force (CPSTF) recommends text messaging interventions to improve medication adherence among patients with chronic diseases. "Health Information Technology: Text Messaging Interventions for Medication Adherence Among Patients with Chronic Diseases" is available at https://www.thecommunityguide.org/findings/ health-information-technology-text-messaging-medicationadherence-chronic-disease.

Established in 1996 by the U.S. Department of Health and Human Services, the CPSTF is an independent, nonfederal panel of public health and prevention experts whose members are appointed by the director of CDC. The CPSTF provides information for a wide range of persons who make decisions about programs, services, and other interventions to improve population health. Although CDC provides administrative, scientific, and technical support for the CPSTF, the recommendations developed are those of the task force and do not undergo review or approval by CDC. 